\title{
BI-ORTHOGONAL FILTER BANKS WITH DIRECTIONAL VANISHING MOMENTS
}

\author{
Arthur L. da Cunha and Minh N. Do \\ University of Illinois at Urbana-Champaign \\ Department of Electrical and Computer Engineering \\ Coordinated Science Laboratory \\ Urbana, IL 61801 \\ Email:\{cunhada,minhdo\}@uiuc.edu
}

\begin{abstract}
In this paper we study 2-D nonseparable filter banks that annihilate information along a certain discrete direction. This is done by having filters with directional vanishing moments (DVM). We study the approximation property of such filters and the design problem providing conditions for its solvability. In particular we completely characterize the solution and propose a design procedure utilizing the mapping technique. Nonlinear approximation experiments with the contourlet transform indicate that compared with the traditional filters, the new filters designed with DVM provide gains in SNR and visual quality due to their short size.
\end{abstract}

\section{INTRODUCTION}

Recently there has been a wide interest in image representations that efficiently handle geometric structure. This comes from the recognition that wavelets essentially fail to take advantage of geometric regularity, a common feature in natural images. In 1-D however, wavelets are known to be optimal in approximating piecewise smooth signals, a feature that is attained by the presence of vanishing moments in the transform. It is thus natural to ask the question of which equivalent feature would be responsible for an optimal approximation of piecewise smooth images.

In [1] the contourlet transform is proposed as a means to fix the failure of wavelets in handling geometry. It is shown that nonlinear approximation using contourlets can achieve the optimal approximation rate for piecewise $C^{2}$ images. This however relies on the presence of directional vanishing moments in the contourlet frame element. This condition can be stated in terms of a filter design constraint.

In this paper we study two channel filter banks with DVM. Two-channel filters are attractive since they are simpler to design and can be used in a tree structure to generate more complicated systems such as the directional filter bank [2]. Our goal is to impose directional vanishing moments in the contourlet basis function without resorting to long filters. That is, we attempt to cancel direction information using DVM's instead of good frequency selectivity, thus working with shorter filters and avoiding the Gibbs phenomena. Potential applications of the filters designed in this work are in the contourlet transform of [1], the CRISP-contourlet system [3] and directionlets [4].

\footnotetext{
Thanks to CAPES and NSF for funding.
}

Notation: Throughout the paper we use boldface and capital boldface characters to represent 2 -D vectors and $2 \times 2$ matrices respectively. Thus, a discrete $2-\mathrm{D}$ signal is denoted by $x[\mathbf{n}]$ where $\mathbf{n}=\left(n_{1}, n_{2}\right)^{T}$. The 2-D $z$-transform of a signal $x[\mathbf{n}]$ is denoted by $X(\mathbf{z})$, where it is understood that $\mathbf{z}$ is short hand for $\left(z_{1}, z_{2}\right)$. If $\mathbf{u}=\left(u_{1}, u_{2}\right)^{T}$ is a vector in $\mathbb{Z}^{2}$, then we denote $\mathbf{z}^{\mathbf{u}}=z_{1}^{u_{1}} z_{2}^{u_{2}}$ whereas, $\mathbf{z}^{\mathbf{S}}=\mathbf{z}^{\mathbf{s}_{1}} \mathbf{z}^{\mathbf{s}_{2}}$ with $\mathbf{s}_{1}$ and $\mathbf{s}_{2}$ being the columns of the matrix $\mathbf{S}$. We also denote the modulation vector $\mathbf{W}_{\mathbf{S}}^{\mathbf{k}}:=e^{j 2 \pi \mathbf{S k}}$ for a $2 \times 2$ matrix $\mathbf{S}$ and an integer vector $\mathbf{k}$.

\section{DIRECTIONAL EIGEN-SIGNALS}

Before we begin, let us formally define directional vanishing moments.

Definition 1 Let $C(\mathbf{z})$ be a discrete filter and $\mathbf{u}=\left(u_{1}, u_{2}\right)^{T}$ a 2-D vector of co-prime integers. We say $C(\mathbf{z})$ has a DVM of order $d$ along direction $\theta=\tan ^{-1} \frac{u_{2}}{u_{1}}$ if it factors as

$$
C\left(z_{1}, z_{2}\right)=\left(1-z_{1}^{u_{1}} z_{2}^{u_{2}}\right)^{d} R\left(z_{1}, z_{2}\right) .
$$

For contourlets, the filter $C(\mathbf{z})$ is a composite one, which involves the Laplacian pyramid filters and the polyphase components of the directional filters [1].

In view of the previous definition and the 1-D case, it is natural to ask what signal would be annihilated (i.e., completely filtered out) by the filter in (1). Such signal is an eigen-signal of the complementary branch of a two-channel FB where $C\left(z_{1}, z_{2}\right)$ is an analysis filter. In fact, those signals can be related to samples of polynomial surfaces separated by one or more edge discontinuities along the direction of the vanishing moment as the next theorem shows.

Theorem 1 Suppose that $x[\mathbf{n}]$ is the signal obtained by sampling a continuous time signal $x_{c}(\mathbf{t})$, that is,

$$
x[\mathbf{n}]=x_{c}(\Delta T \mathbf{n})
$$

where $x_{c}(\mathbf{t})$ is a piecewise polynomial of degree less than $d$ separated by lines of slope $\theta=\tan ^{-1} \frac{u_{2}}{u_{1}}$. Then $x[\mathbf{n}]$ is annihilated by $C(\mathbf{z})$.

Proof: See [5]. Figure 2 illustrates a typical signal described in Theorem 1 and its filtered version. The filter has a DVM along the edge discontinuity direction. 

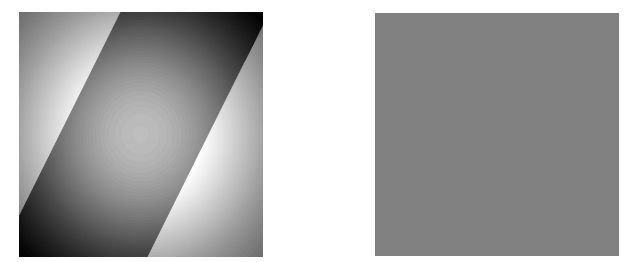

Fig. 1. Illustration of line zero moments as an edge annihilator. The piecewise polynomial image in (a) was filtered with a 2-D filter $H\left(z_{1}, z_{2}\right)=\left(1-z_{1} z_{2}^{2}\right)^{3}$. The output image (b) has no edges.

\section{TWO-CHANNEL FILTER BANKS WITH DIRECTIONAL VANISHING MOMENTS}

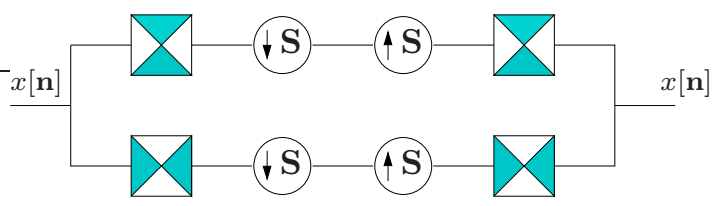

Fig. 2. Two-channel Filter Bank.

A typical 2-channel filter bank is the Fan filter bank illustrated in Figure 2. For the FIR case with an arbitrary down-sampling matrix $\mathbf{S}$, the synthesis/analysis filters are related by

$\left(H_{1}(\mathbf{z}), G_{1}(\mathbf{z})\right)=\left(\mathbf{z}^{\mathbf{k}_{1}} G_{0}\left(\mathbf{W}_{\mathbf{S}^{-T}}^{\mathbf{k}_{1}} \circ \mathbf{z}\right), \mathbf{z}^{-\mathbf{k}_{1}} H_{0}\left(\mathbf{W}_{\mathbf{S}^{-T}}^{\mathbf{k}_{1}} \circ \mathbf{z}\right)\right)$ where $(\cdot)^{T}$ stands for vector transposition, "o" denotes direct product of vector entries and $\mathbf{k}_{1}$ is the nonzero integer vector in the set $\mathcal{N}(\mathbf{S}):=\left\{\mathbf{S}^{T} x, x \in[0,1) \times[0,1)\right\}([6]$ pp. 575). The analysis/synthesis relation gives the perfect reconstruction condition:

$$
H_{0}(\mathbf{z}) G_{0}(\mathbf{z})+H_{0}\left(\mathbf{W}_{\mathbf{S}^{-T}}^{\mathbf{k}_{1}} \circ \mathbf{z}\right) G_{0}\left(\mathbf{W}_{\mathbf{S}^{-T}}^{\mathbf{k}_{1}} \circ \mathbf{z}\right)=2 .
$$

Thus, our problem consists in designing filters $H_{0}(\mathbf{z})$ and $G_{0}(\mathbf{z})$ such that the above PR condition is met and in addition the filters have the form in Definition 1, that is

$$
H_{0}(\mathbf{z})=\left(1-z_{1}^{u_{1}} z_{2}^{u_{2}}\right)^{N_{a}} R_{H_{0}}(\mathbf{z})
$$

and

$$
G_{0}(\mathbf{z})=\left(1-z_{1}^{u_{1}} z_{2}^{u_{2}}\right)^{N_{s}} R_{G_{0}}(\mathbf{z})
$$

where $N_{a}$ and $N_{s}$ denote the order of the DVM in the analysis and synthesis parts respectively. Substituting these filters in (2) we obtain the design equation

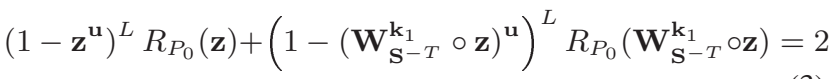

where $R_{P 0}(\mathbf{z}):=R_{H_{0}}(\mathbf{z}) R_{G_{0}}(\mathbf{z})$ and $L=N_{a}+N_{s}$.

It can be shown [5] that an FIR filter $R_{P_{0}}(\mathbf{z})$ satisfying the above equation exists if and only if $\mathbf{u}^{T} 2 \mathbf{S}^{-T} \mathbf{k}_{1}$ is an odd integer. If that is the case, the following proposition greatly simplifies the problem.

Proposition 1 Consider the filter equation (3) with $\mathbf{u}$ such that $\mathbf{u}^{T} 2 \mathbf{S}^{-T} \mathbf{k}_{1}$ is odd. Then there exists a unimodular ${ }^{1}$ integer matrix

\footnotetext{
${ }^{1}$ A square matrix is unimodular if its determinant is equal to one.
}

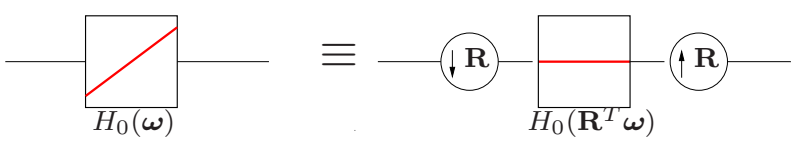

Fig. 3. Change of variable is equivalent to a pre/post re-sampling operation plus filtering with modified filter.

$\mathbf{R}$ such that if $R(\mathbf{z})$ solves (3) then $\tilde{R}(\mathbf{z})=R\left(\mathbf{z}^{\mathbf{R}}\right)$ solves

$$
\left(1-z_{1}\right)^{L} \tilde{R}(\mathbf{z})+\left(1+z_{1}\right)^{L} \tilde{R}\left(\mathbf{W}_{\mathbf{R}^{-1} \mathbf{S}^{-T}}^{\mathbf{k}_{1}} \mathbf{z}\right)=2
$$

Conversely, if $\tilde{R}(\mathbf{z})$ is a solution to (4), then there exists a matrix $\mathbf{R}$ such that $R(\mathbf{z})=\tilde{R}\left(\mathbf{z}^{\mathbf{R}^{-1}}\right)$ is a solution to (3).

The previous Proposition asserts that it suffices to consider the design of filters with DVM's along the horizontal direction. It can be interpreted in terms of pre/post re-sampling operations as Figure 3 illustrates. In addition, we need to consider (4) with only two possible forms for $\tilde{R}\left(\mathbf{W}_{\mathbf{R}^{-1} \mathbf{S}^{-T}}^{\mathbf{k}_{1}} \mathbf{z}\right)$, namely $\tilde{R}\left(-z_{1}, z_{2}\right)$ or $\tilde{R}\left(-z_{1},-z_{2}\right)$ corresponding to the rectangular and quincunx lattices. The other possible lattice is the transpose of the rectangular. The next proposition provides a complete characterization of the solution to (4).

Proposition 2 Let $a \in\{1,-1\}$, then an FIR filter $\tilde{R}\left(z_{1}, z_{2}\right)$ is solution to the equation

$$
\left(1-z_{1}\right)^{L} \tilde{R}\left(z_{1}, z_{2}\right)+\left(1+z_{1}\right)^{L} \tilde{R}\left(-z_{1}, a z_{2}\right)=2
$$

if and only if it has the form

$$
\tilde{R}\left(z_{1}, z_{2}\right)=R_{L}\left(z_{1}\right)+\left(1+z_{1}\right)^{L} R_{o}\left(z_{1}, z_{2}\right)
$$

with $R_{L}\left(z_{1}\right)$ being a univariate solution given explicitly by

$$
R_{L}\left(z_{1}\right)=\sum_{i=0}^{L-1}\left(\begin{array}{c}
L+i-1 \\
L-1
\end{array}\right) 2^{-(L+i-1)}\left(1+z_{1}\right)^{i}
$$

and $R_{o}(\mathbf{z})$ satisfying

$$
R_{o}\left(z_{1}, z_{2}\right)+R_{o}\left(-z_{1}, a z_{2}\right)=0
$$

Remarks:

1. The above Proposition is akin to its 1-D counterpart which is used to construct compactly supported wavelets (see e.g., [7]). The distinction occurs in the higher order term which now can be any two-dimensional function satisfying (8). This higher order term will make the filter a truly two dimensional one, meaning a filter with a nonseparable support. Moreover, this higher order term can be used to control the shape of the 2-D frequency response.

2. This already provides a class of biorthogonal filters with DVM in which each one of the filters in the analysis and synthesis is a degenerate 1-D solution. Thus, the filters obtained as in Proposition 2 can be seen as a 2-D generalization of the bi-orthogonal spline wavelet filters of [7].

Proposition 2 provides a complete characterization of the product filter $H_{0}(\mathbf{z}) G_{0}(\mathbf{z})$ for a filter bank having DVM's. To obtain the filters one needs to factorize this product which is difficult in multiple dimensions. Next we show a way to overcome this need by using transformation of variable. 


\section{DESIGN}

One successful method for multidimensional filter design is the transformation of variable or mapping technique discussed in [8]. This method has the desirable feature of easy control of the frequency and phase responses. It consists in replacing the complex variable $z$ in a 1-D biorthogonal pair of filters, or some 1-D function of it, by a function defined on the $\mathbb{C}^{2}$ plane. Specifically, we may write the 1-D filters as $H_{0}^{(1 \mathrm{D})}(f(z)), G_{0}^{(1 \mathrm{D})}(f(z))$ and then use the mapping $f(z) \mapsto M(\mathbf{z})$, the same applying for the remaining filters. It is not hard to see that perfect reconstruction condition will be kept provided [8]

$$
f(z)=-f(-z), \text { and, } M(\mathbf{z})=-M\left(\mathbf{W}_{\mathbf{S}^{-T}}^{\mathbf{k}_{1}} \circ \mathbf{z}\right) .
$$

For FIR solutions, one typically has $f(z)=z+z^{-1}$ for zerophase filters or $f(z)=z$ for filters consisting of only positive powers of $z$. Notice that in both cases, one cannot have $G_{0}^{(1 \mathrm{D})}(z)=$ $H_{0}^{(1 \mathrm{D})}\left(z^{-1}\right)$.

In the context of DVM, the goal is to devise a mapping function $M(\mathbf{z})$ such that each of the 2-D filters $H_{0}(\mathbf{z}):=H_{0}^{(1 \mathrm{D})}(M(\mathbf{z}))$, $G_{0}(\mathbf{z}):=G_{0}^{(1 \mathrm{D})}(M(\mathbf{z}))$ has a given number of $\left(1-z_{1}\right)$ factors. In addition we require $M(\mathbf{z})$ so that perfect reconstruction is kept after mapping. The next proposition shows an explicit form of the required mapping function.

Proposition 3 Let $H_{0}^{(I D)}(f(z)), G_{0}^{(I D)}(f(z))$ be a bi-orthogonal 1-D filter pair and $a \in\{1,-1\}$. Suppose $M(\mathbf{z})$ is an FIR mapping function such that

$$
M\left(z_{1}, z_{2}\right)=\left(1-z_{1}\right)^{L} \tilde{R}\left(z_{1}, z_{2}\right)+c_{0}
$$

where $c_{0}$ is such that $H_{0}^{(I D)}(f(z))$ has a factor $\left(f(z)-c_{0}\right)^{N_{a} / L}$, $G_{0}^{(I D)}(f(z))$ has a factor $\left(f(z)-c_{0}\right)^{N_{s} / L}$, and $\tilde{R}\left(z_{1}, z_{2}\right)$ satisfies the valid mapping equation:

$$
\left(1-z_{1}\right)^{L} \tilde{R}\left(z_{1}, z_{2}\right)+\left(1+z_{1}\right)^{L} \tilde{R}\left(-z_{1}, a z_{2}\right)=2 c_{0} .
$$

Then

1. $H_{0}(\mathbf{z}), G_{0}(\mathbf{z})$ form a bi-orthogonal pair, i.e., they satisfy

$$
H_{0}\left(z_{1}, z_{2}\right) G_{0}\left(z_{1}, z_{2}\right)+H_{0}\left(-z_{1}, a z_{2}\right) G_{0}\left(-z_{1}, a z_{2}\right)=2 .
$$

2. The mapped filters factor as

$$
H_{0}(\mathbf{z})=\left(1-z_{1}\right)^{N_{a}} R_{H_{0}}(\mathbf{z}), \quad G_{0}(\mathbf{z})=\left(1-z_{1}\right)^{N_{s}} R_{G_{0}}(\mathbf{z}) .
$$

The previous proposition together with the characterization of the mapping function of Proposition 2 allows for an easy design methodology that can be summarized in the following steps:

Step 1 Design a 1-D filter pair $H_{0}^{(1 \mathrm{D})}(f(z)), G_{0}^{(1 \mathrm{D})}(f(z))$ with $N_{a}$ and $N_{s}$ zeros at some point $c_{0} \in \mathbb{C}$. If solution needs to be FIR, then constrain the filters to have positive powers of $f(z)$ only.

Step 2 If necessary, perform a change of variables to convert the problem to the form in (4).

Step 3 Let $M(\mathbf{z})=\left(1-z_{1}\right)^{L} \tilde{R}(\mathbf{z})+c_{0}$ with $\tilde{R}(\mathbf{z})=\tilde{R}_{L}\left(z_{1}\right)+$ $\left(1+z_{1}\right)^{L} \tilde{R}_{o}\left(z_{1}, z_{2}\right)$ and $\tilde{R}_{o}\left(z_{1}, z_{2}\right)=-\tilde{R}_{o}\left(-z_{1}, a z_{2}\right)$
Step 4 Substitute $f(z) \mapsto M(\mathbf{z})$ in the 1-D filters and obtain the 2-D ones.

The next example illustrates how a typical design is carried out. Refer to [5] for more details.

Example 1 In order to get a solution with four directional vanishing moments (for the Quincunx lattice), we use the minimal degree complementary filter to $(1-f(z))^{4}$, which from (7), gives the product filter

$P^{(I D)}(f(z))=\frac{1}{16}\left(16-29 f(z)+20 f(z)^{2}-5 f(z)^{3}\right)(1-f(z))^{4}$,

where we let $f(z)=\left(z+z^{-1}\right) / 2$ to obtain zero-phase solutions. We factor the above polynomial assigning two zeros for one filter and one for the other. Moreover, the fourth order zero at $f(z)$ is equally split between $H_{0}^{(I D)}(f(z))$, and $G_{0}^{(I D)}(f(z))$. For the mapping function we choose it to have second order zero moment term. The mapping function we use is

$$
M\left(z_{1}, z_{2}\right)=\left(2+z_{1}+z_{1}^{-1}\right) R\left(z_{1}, z_{2}\right)-1
$$

where to guarantee that the map satisfies the valid mapping condition (10) we pick

$$
R\left(z_{1}, z_{2}\right)=1+\left(2-z_{1}-z_{1}^{-1}\right) R_{o}\left(z_{1}, z_{2}\right)
$$

For simplicity we choose $R_{o}\left(z_{1}, z_{2}\right)=\alpha\left(z_{2}+z_{2}^{-1}\right)$. With $\alpha=0$ we set the 9-7 filters. Figure 4 illustrate the analysis/synthesis pair frequency response when we set $\alpha=\sqrt{2}$.
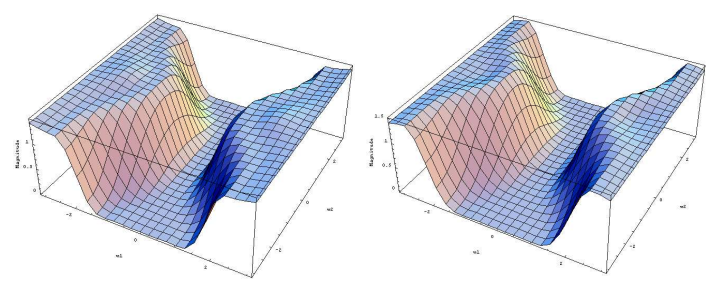

Fig. 4. Frequency response of example analysis and synthesis filters designed with 4-th order directional vanishing moment.

\section{EXPERIMENTS}

In order to illustrate the applicability of the directional vanishing moment filters proposed, we perform an experiment in which we replace the DFB filters of the contourlet construction with the filters designed with zero moments, following the discussion in [5]. In order to assess the filters performance, we reconstruct the detail subspace $W_{j}$ with the $N$ largest coefficients and compute the corresponding SNR. The directional expansion tree we use in each scale is one that leads to a maximum number of distinct DVMs and is the same for all test images, hence the expansion is fixed. The analysis filter is zero-phase with $7 \times 13$ coefficients and the synthesis with $9 \times 17$, also zero-phase. As a comparison, we use the quincunx/fan filters of [9] (PKVA), where the analysis filter has $23 \times 23$ taps and the synthesis $46 \times 46$. 


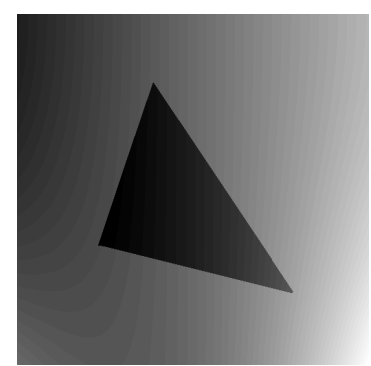

(a)

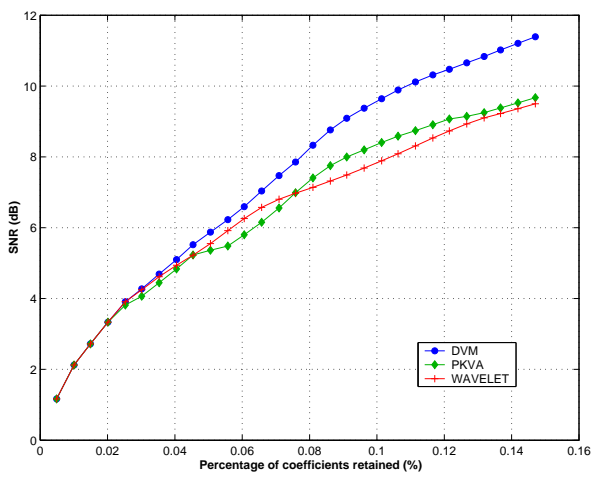

(b)

Fig. 5. NLA Approximation on the detail subspace. (a) Synthetic piecewise polynomial image. (b) SNR curves for the Synthetic image.

Figure 5 displays the curve of a piecewise polynomial image. For this synthetic image, a significant improvement is observed. Such improvement is due to the fact that the synthetic image has directional information in a very small set of directions, which, due to DVM's, are well represented in the expansion. For natural images the DVM filters render a slightly better performance than the PKVA filters. However, because the DVM filters are considerably shorter, we observe less ringing artifacts when compared against the PKVA filters, even when both give similar SNR. Figure 6 shows the "Peppers" image reconstructed with 2048 coefficients using both of the filters. As can be seen, the image reconstructed with the DVM filters exhibit less ringing artifacts.

\section{CONCLUSION}

We have studied 2-channel biorthogonal filter banks in which one filter bank channel kills information along a prescribed direction by means of directional vanishing moments. Such filters have proven to be useful as building blocks for more complicated decomposition structures such as the contourlet expansion. We studied the DVM filter bank design problem and provided a complete characterization of the product filter. Using the mapping design methodology, we proposed a design procedure in which the mapping filter can be calculated explicitly. Experimental results indicate that filters with DVM's can be as good as filters designed with frequency response as a primary criteria and, in some cases, yield better results. In addition, because the filters are short, the Gibbs phenomena is considerably reduced.

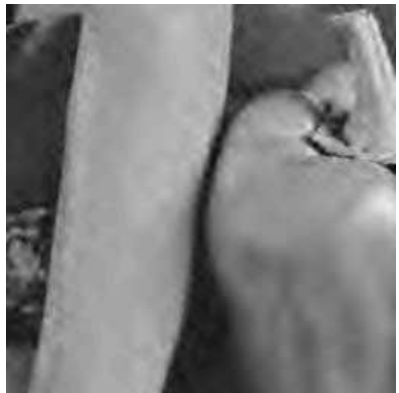

(a) Old PKVA filters

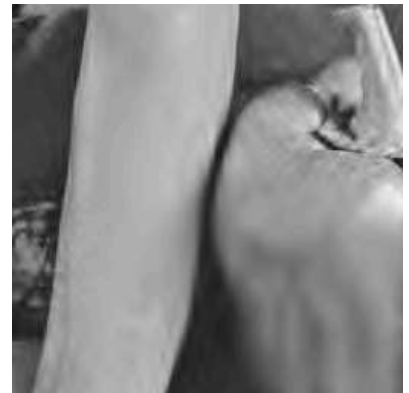

(b) New DVM filters
Fig. 6. "Peppers" Image reconstructed with 2048 coefficients. (a) Old PKVA filters, (b) New DVM filters. The image on the right shows less ringing artifacts.

\section{REFERENCES}

[1] M. N. Do and M. Vetterli, "The contourlet transform: An efficient directional multiresolution image representation," IEEE Trans. Img. Processing, to appear.

[2] R. H. Bamberger and M. J. T. Smith, "A filter bank for the directional decomposition of images: Theory and design," IEEE Trans. Signal Processing, vol. 40, no. 4, pp. 882-893, April 1992.

[3] Y. Lu and M. Do, "Crisp-contourlet: a critically sampled directional multiresolution representation," in Proc. SPIE Conf. on Wavelets $X$, San Diego, CA, Aug. 2003.

[4] V. Velisavljević, B. Beferull-Lozano, and M. Vetterli, "Directionlets: regular multi-directional wavelet bases with one-dimensional filtering," ICIP, 2004

[5] A. L. da Cunha and M. N. Do, "Biorthogonal two-channel filter banks with directional vanishing moments: Characterization, design and applications," IEEE Trans. Img. Proc., submitted.

[6] P. Vaidyanathan, Multirate Systems and Filterbanks. Prentice Hall, 1993.

[7] I. Daubechies, Ten Lectures on Wavelets. Philadelphia, PA: SIAM, 1992.

[8] D. B. H. Tay and N. G. Kingsbury, "Flexible design of multidimensional perfect reconstruction FIR 2-band filters using transformation of variables," IEEE Trans Img Proc., vol. 2, no. 4, pp. 466-480, October 1993.

[9] S.-M. Phoong, C. W. Kim, P. P. Vaidyanathan, and R. Ansari, "A new class of two-channel biorthogonal filter banks and wavelet bases," IEEE Trans. Sig. Proc., vol. 43, no. 3, pp. 649-661, March 1995. 